\title{
EFFICIENCY ANALYSIS OF LOW-DENSITY PARITY CHECK CODES USING EXIT CHARTS
}

\author{
Evgeny I. Mirokhin, Evgeny A. Likhobabin, Alexey A. Ovinikov, Vladimir V. Vityazev \\ Department of Telecommunications and Radio Engineering of Ryazan State \\ Radio Engineering University \\ Gagarina Str. 59/1, Ryazan 390005, Russia
}

The paper is received on April 25, 2019

\begin{abstract}
At present, iterative decoding schemes are gaining popularity, such as: turbo codes, low density parity checks codes and polar codes. In this paper, an analysis of energy efficiency was performed using extrinsic information transfer function for low density parity check codes. The extrinsic information transfer functions charts were obtained, allowing us to estimate the energy efficiency of the code without invoking Monte Carlo simulation.
\end{abstract}

Keywords: component, formatting, ldpc, exit charts.

\section{Introduction}

Low density parity check codes are a class of linear block codes that have excellent performance. Like any linear block code, the LDPC code can be represented by a parity check matrix $H$. However, as the name suggests, the main difference between LDPC codes and conventional linear block codes is the LDPC or, in other words, the verification matrix $\mathrm{H}$ of such code contains an extremely small number of ones. The density of the units should be low enough only to enable the application of an efficient, from the point of view of the computational cost, iterative decoding algorithm. The low density of checks in LDPC codes makes it possible to apply iterative decoding, whose efficiency, for bit error probabilities of practical interest, is usually very close to optimal, and computational costs are proportional to the length of the decoded code $[1,6]$. The Tanner graph of the LDPC code provides a complete view of the code and helps in describing the decoding algorithms. To achieve this result, codes of a large length are required, which require large computational power for modeling, which is not always possible. However, there are methods that allow us 
to analyze the quality of the code without Monte Carlo simulation. In 1981, RM Tanner proposed [7] to use for the description of LDPC codes bipartite non-directional graphs, which were also known as Tanner graph, the nodes of which can be divided into two types with edges connecting nodes of different types, which is shown on the figure 1. Nodes are called information and check, which we denote as $V_{N}$ and $C_{C N}$, respectively. Each of the nodes of the graph can be considered as a local decoder, and each edge is a bus that transmits messages from the node to the neighbor. The transmitted messages are reliability information, typically LLR (log-likelihood ratio, L-value) for bits corresponding to the information nodes. So, each of the local decoders processes all messages $L_{i}$, coming from neighboring nodes, and sends back the result obtained for each of the neighbors. The efficiency of the iterative decoder depends on a number of structural features of the Tanner graph of the code used.
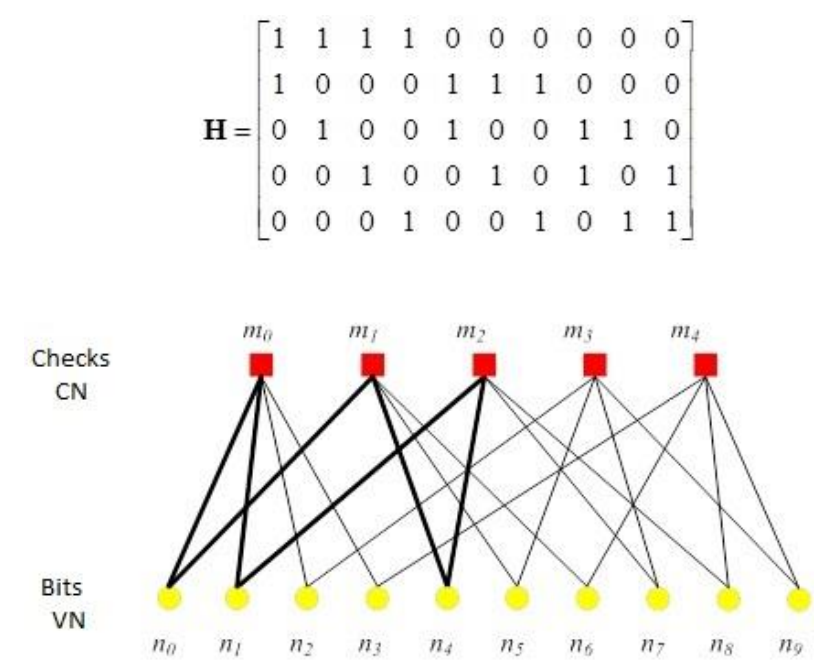

Fig. 1. Parity check matrix and Tanner graph of LDPC code.

\section{Iterative decoding}

\section{A. Iterative decoding scheme}

Iterative decoding of LDPC is a powerful tool for obtaining energy efficiency close to the Shannon's limit. LDPC can be divided into regular and irregular. A code is called $\left(d_{v}, d_{c}\right)$-regular if every column and every row of its parity check matrix contains $d_{v}$ and $d_{c}$ non-zero elements, while the encoding rate is described by the formula $R \geq 1-d_{v} / d_{c}$, otherwise the code is called irregular. The set of variable and 
check nodes is denoted by the decoder of the information nodes (variable-node decoder - VND) and the decoder of the check nodes (check-node decoder - $C N D$ ). Iterative decoding is the exchange of messages between $V N D$ and ${ }_{C N D}$. The structure of the decoder is shown in Figure 2.

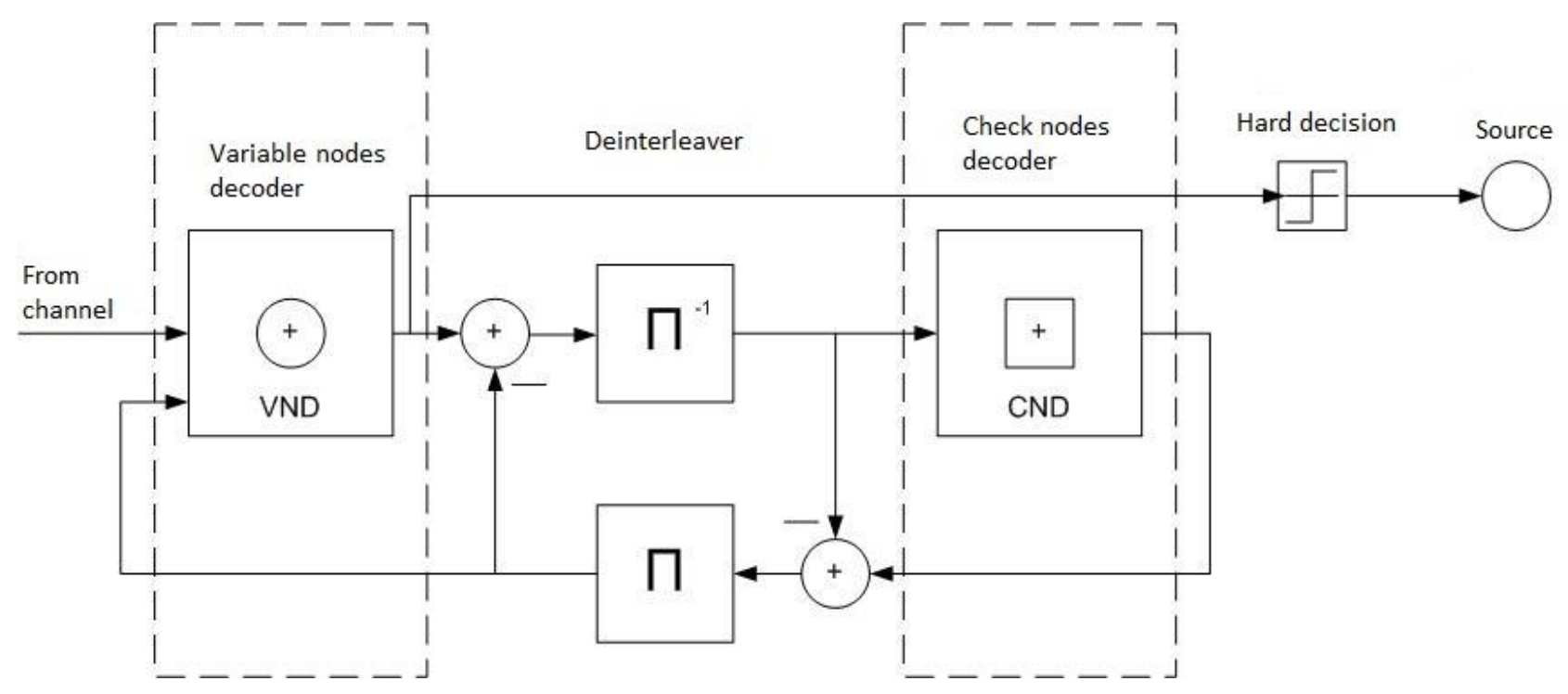

Fig. 2. Iterative decoding scheme.

\section{B. Mutual information for VND and CND}

VND, which is a repetition code, is treated as extrinsic decoder, since it has a direct connection to the communication channel, and the CND, the parity check code, does not have direct access to the communication channel and its input is the output of the repetition code. VND converts the channel output, as well as $L_{a p p s, C N D}$-values to $L_{\text {apss, }, V D D}$-values, which in turn serve as $L_{\text {aprior }}$-values for CND. To analyze the operation of the decoder, it is convenient to use the dependencies of the transfer function of external information characterizing the operation of the decoder [8]. To construct such dependencies, it is necessary to introduce such a notion as mutual information $I_{A}$ between transmitted bits and $L$-values, having the form:

$$
I_{A}=\frac{1}{2} \sum_{x=-1,1} \int_{-\infty}^{+\infty} p_{a}(\xi \mid X=x) \ln \frac{2 p_{a}(\xi \mid X=x)}{p_{a}(\xi \mid X=-1)+p_{a}(\xi \mid X=1)},
$$

where, $\xi$ - received value from the channel, $X$-value with which is considered the $\log$ likelihood ratio. 
$I_{A}$ means $L$-values based on a Gaussian distribution, so the mean $\mu_{A}$ is calculated as:

$$
\mu_{A}=\frac{\sigma_{A}^{2}}{2},
$$

and the probability density function of $L$-values is represented:

$$
p_{a}(\xi \mid X=x)=\frac{e^{-\left(\left(\xi-\left(\sigma_{A}^{2} / 2\right)-x\right)^{2}\right) / 2 \sigma_{A}^{2}}}{\sqrt{2 \pi} \sigma_{A}},
$$

it can be shown [10] that when substituting (2) and (3) in (1), we obtain the following entry for mutual information:

$$
I_{A}\left(\sigma_{A}\right)=1-\int_{-\infty}^{+\infty} \frac{-\left(\left(\xi-\left(\sigma_{A}^{2} / 2\right)-x\right)^{2}\right) / 2 \sigma_{A}^{2}}{\sqrt{2 \pi} \sigma_{A}} \ln \left[1+e^{-\xi}\right] d \xi,
$$

for simplified recording:

$$
J(\sigma)=I_{A}\left(\sigma_{A}=\sigma\right)
$$

where $J(\sigma)$ can take on values:

$$
\lim _{\sigma \rightarrow 0} J(\sigma)=0, \lim _{\sigma \rightarrow \infty} J(\sigma)=1, \sigma>0 \text {. }
$$

The information node receives messages from the interleaver and from the communication channel, decoding them by computing:

$$
L_{i, e x t}=L_{c h}+\sum_{j \neq i} L_{i, a p r i o, V N D},
$$

where $L_{\text {iext }}$ this is the $i$-th outer $L$-value obtained from the information node, $L_{c h}$ the channel $L$-value.

The signal-to-noise ratio in the channel with additive white Gaussian noise, over which the modulated BPSK signal is transmitted, is described by equation $E_{b} / N_{o}=1 / 2 R \sigma_{n}^{2}$. Channel L-value in this case will be [10]: 


$$
L_{c h}=\ln \frac{p(y \mid x=+1)}{p(y \mid x=-1)}=\frac{2}{\sigma_{n}^{2}} y,
$$

where $p(y \mid x)$ conditional probability density function, estimated for $\mathrm{y}$ when $\mathrm{x}$ transmitted.

Denote $X$ and ${ }_{Y}$ as random values that identify the channel's input and output. Then the variance of $L_{c h}$ is due to the value of $X_{X}[10]$ :

$$
\sigma_{c h}^{2}=\frac{4}{\sigma_{n}^{2}}=8 R \frac{E_{b}}{N_{o}}
$$

\section{Simulation results}

To plot the extrinsic information transfer function it is necessary to model the change in the value of the channel $L$-values, thereby obtaining various output external values from the output $V N D$. Formulas for plotting graphs of external information functions for an information node have the form:

$$
\begin{gathered}
I_{E, V N D}\left(I_{A}, d_{v}, \frac{E_{b}}{N_{o}}, R\right)=J\left(\sqrt{\left(d_{v}-1\right)\left[J^{-1}\left(I_{A}\right)\right]^{2}+\sigma_{c h}^{2}}\right), \\
I_{E, C N D}\left(I_{E}, d_{c}\right) \approx 1-J\left(\sqrt{d_{c}-1} \cdot J^{-1}\left(1-I_{A}\right)\right),
\end{gathered}
$$

where function $J(\cdot)$ is represented by the formula (4). EXIT charts for the check node and variable node are shown in Figures 3 and 4 with different degree and speed $R=1 / 2$ and signal-to-noise ratio $E_{b} / N_{o}=1 \mathrm{~dB}$.

C. EXIT charts for irregular codes

For irregular codes, the functions change their appearance due to the irregularity of the distribution of weights in the code and is described with the help of weight polynomials of variable degrees and check degrees having the form:

$$
\lambda(x)=\sum_{d=1}^{d_{\text {vmax }}} \lambda_{d} x^{d-1}, p(x)=\sum_{d=1}^{d_{\text {cmax }}} p_{d} x^{d-1},
$$

where $\lambda_{d}$ and $p_{d}$ the proportion of columns and rows in the check matrix, of weight $d, d_{c \max }, d_{v \max }$. 


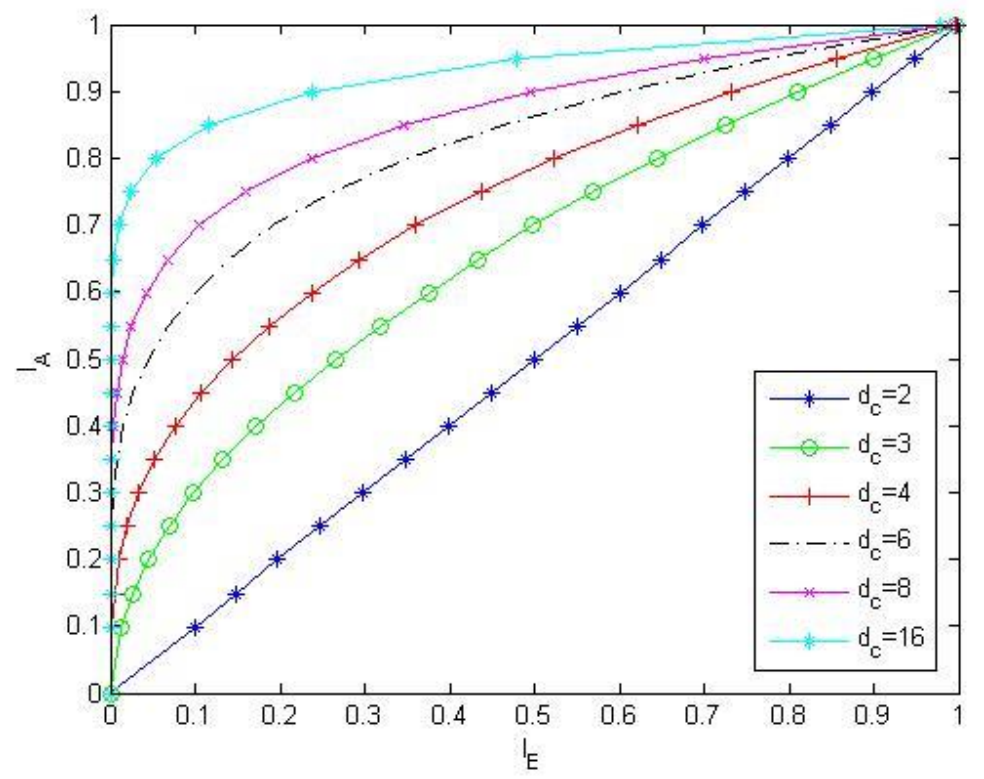

Fig. 3. EXIT charts for check node.

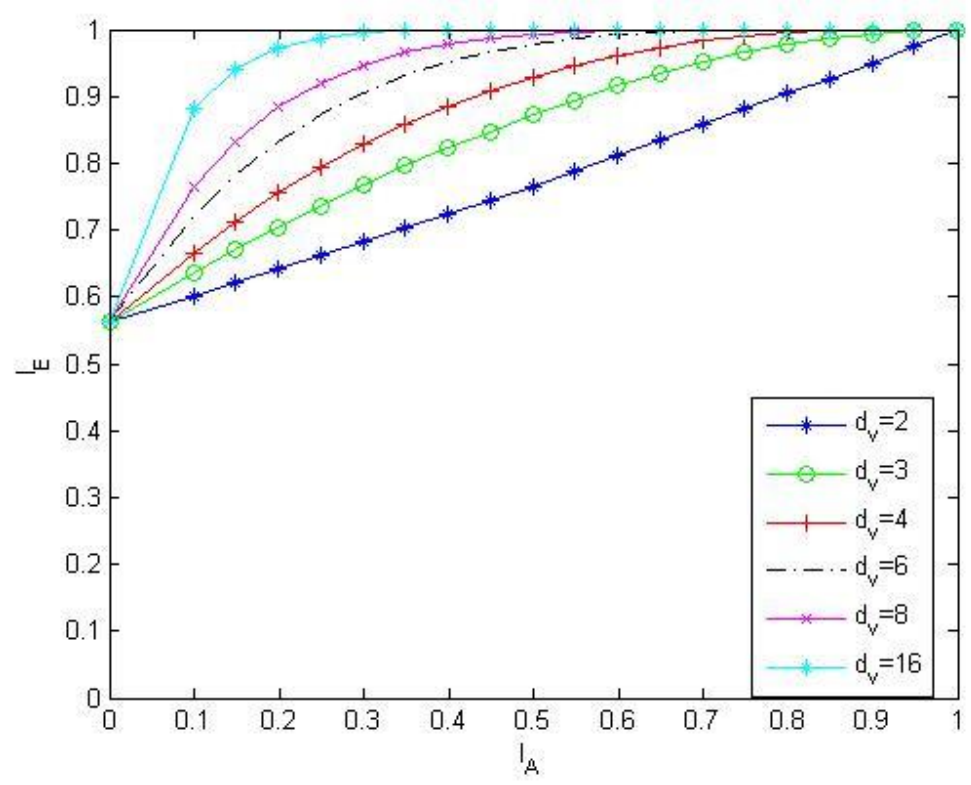

Fig. 4. EXIT charts for variable node.

To verify the obtained algorithm for finding the threshold, an irregular code was taken from the source [9], since irregular codes are of greater interest due to better energy efficiency. Using the algorithm of Gaussian approximation in the source [9], the threshold for the presented code was equal to $E_{b} / N_{\text {olim }}=0.7299$. When calculating the threshold using the proposed method, the graphs converge to unity, which means correct decoding of the codeword. When conducting an experiment with a value less than the threshold value $E_{b} / N_{o}$ decoding becomes impossible, as is confirmed in [9]. The code distribution looks like: 


$$
\begin{aligned}
& \lambda(x)=0.3266 x+0.11960 x^{2}+0.18393 x^{3}+0.36988 x^{4} \\
& p(x)=0.78555 x^{5}+0.21455 x^{6}
\end{aligned}
$$

EXIT charts for threshold $E_{b} / N_{o \text { lim }}=0.7299$ and $E_{b} / N_{o}=0.2299$ is represented on Figure 5 and 6.

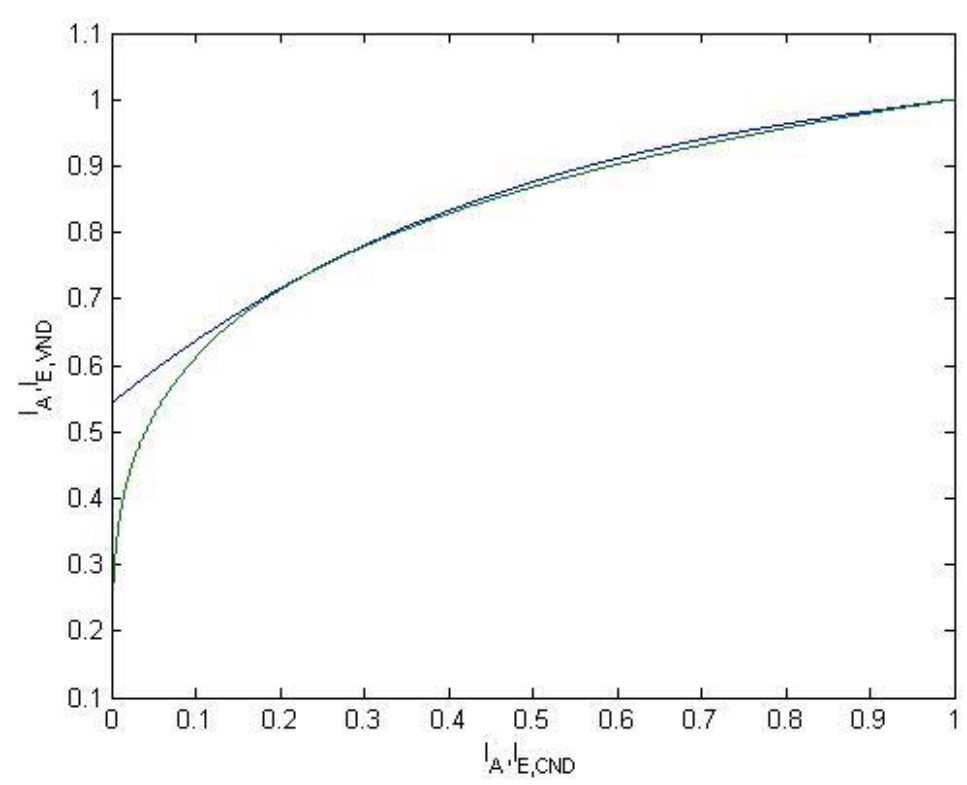

Fig. 5. EXIT chatrs with threshold $E_{b} / N_{\text {olim }}=0.7299$

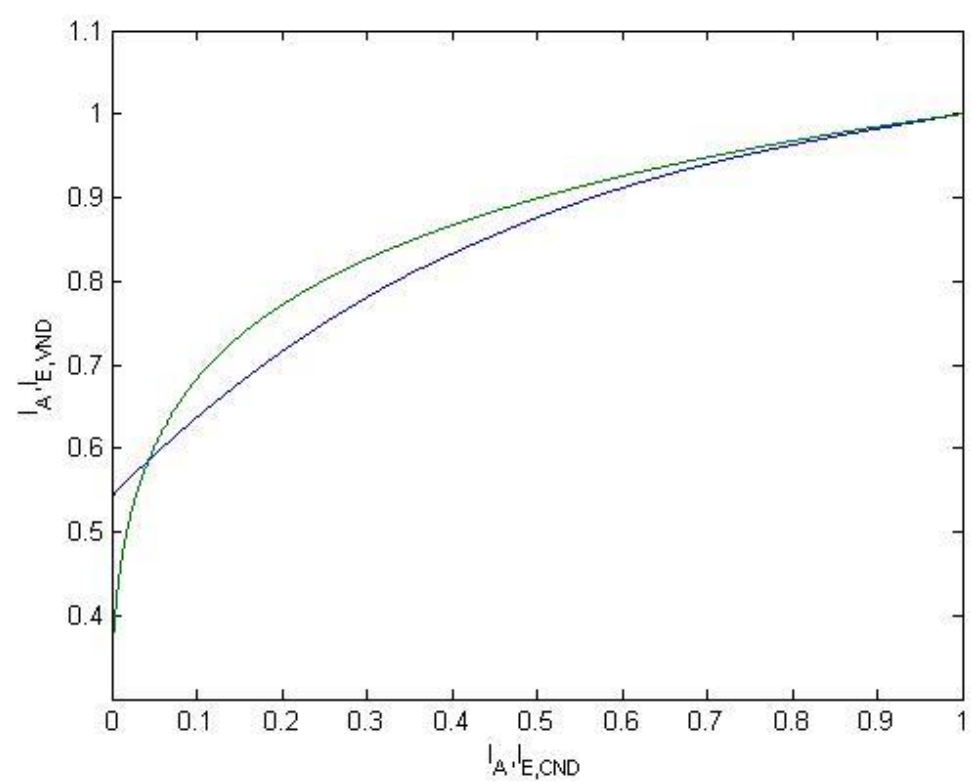

Fig. 6. EXIT charts with threshold $E_{b} / N_{o}=0.2299$

Figure 5 shows that the curves do not intersect, which means that it is possible to properly decode the received codeword, in contrast to Figure 6, where the curves 
intersect, because decoders start to introduce false information into the codeword and proper decoding becomes impossible.

\section{Conclusion}

In this paper, algorithm with EXIT charts analysis was proposed for calculating the thresholds of LDPC code ensembles under sum-product decoding.

The proposed algorithm can be used to calculate the thresholds of LDPC code ensembles. We verified that the thresholds obtained by our algorithm closely coincide with the values computed by density evolution.

This work was supported by Russian Science Foundation under Grant 17-79-20302.

\section{References}

1. Gallager R.G Low-density parity-check codes. Cambridge, MA, M.I.T Press. 1963

2. Wiberg N. Codes and decoding on general graphs. Linkoping University, Sweden, 1996.

3. Davey M.C. Error-correction using low-density parity-check codes. University of Cambridge, 1999.

4. Declercq D., Fossorier M., Bigleri E. Channel coding. Theory, algorithms and applications. Academic press library in mobile and wireless communications, 2014, 667 p.

5. Bahl L., Cocke J., Jelinek F., Raviv J. Optimal decoding of linear codes for minimizing symbol error rate. IEEE Transactions in Information Theory, 1974, Vol. 20, No. 3, pp. 284-287.

6. MacKay D.J.C., Neal R.M. Near Shannon limit performance of low density parity check codes. Electronics Letters, 1996, Vol. 32, pp. 1645-1646

7. Tanner R.M. A recursive approach to low complexity codes. IEEE Transactions in Information Theory, 1981, Vol. 27, No.5, pp. 533-547.

8. T. J. Richardson, A. Shokrollahi, and R. L. Urbanke. Design of capacity approaching low-density parity-check codes. IEEE Trans. Inform. Theory, 2001, Vol. 47, pp. 619-637. 
9. Piming Ma,Kyung Sup Kwak. Thresholds Calculation of LDPC Code Ensembles Using a Novel Gaussian Approximation Algorithm. Proceedings of 2009 IEEE Wireless Communications \& Networking Conference (WCNC'09). Budapest, Hungary, April 05-08, 2009, IEEE Press Piscatway, NJ, USA

10.Urbanke R. Efficient decoding techniques for LDPC codes. Dissertation, Wien. 2007.

\section{For citation:}

Evgeny I. Mirokhin, Evgeny A. Likhobabin, Alexey A. Ovinikov, Vladimir V. Vityazev. Efficiency analysis of low-density parity check codes using EXIT charts. Zhurnal Radioelektroniki - Journal of Radio Electronics. 2019. No.5. Available at http:/jre.cplire.ru/jre/may19/8/text.pdf

DOI $10.30898 / 1684-1719.2019 .5 .8$ 